\title{
Assessment of the Impact of the Motor Transport Complex on the Ecological and Demographic Situation in the Region
}

\author{
Vladimir Gordon ${ }^{1 *}$, Olga Pilipenko ${ }^{1}$, and Tatiana Poturaeva ${ }^{1}$ \\ ${ }^{1}$ Orel State University, 95 Komsomolskaya str., Orel, 302026, Russia
}

\begin{abstract}
The aim of this work is to build a mathematical model of the dynamics and mutual influence of the population and environmental factors - pollution of the atmosphere and surface water bodies - on an urbanized territory. The paper proposes a simulation model of dynamics and interaction of external and internal directions in biosphere-compatible city activities. The external direction of city's activities is removal of resources from the Biosphere and emission of waste polluting water, air and soil into it. The internal direction is the impact on the population: environmental state, health assessment, life expectancy, human potential. Motor transport and objects of the road infrastructure bring the main volume of all waste and emissions polluting the urban environment. The method of forecasting the ecological and demographic situation of an urbanized territory is constructed using a relatively simple, non-linear, with variable coefficients, mathematical model that reflects the interaction of several subsystems: population, pollution of the atmosphere and water bodies.
\end{abstract}

\section{Introduction}

Current demographic tendencies in Russia have a crisis character and are caused by various factors - historical, social, economic, environmental, etc. Since 1990, the process of population decline has been going on in Russia. Its population declined from and 148.3 million in 1990 to 143 million in 2011 [1].

As statistical studies show, there is a fairly strong correlation between levels of air and water pollution and demographic indicators, in particular, the population $[2,3,5]$. Unfavorable environmental conditions affect people's health state and life expectancy. Papers $[4,6]$ presents data demonstrating the relationship between chronic diseases and place of residence: the higher the level of urbanization is, the higher the morbidity, especially in old age, is.

According to the State reports on the state and environmental protection in the Russian Federation, in 136 cities ( $55 \%$ of urban population) air pollution is characterized as high or very high. Emissions of pollutants into the atmosphere from 2000 to 2009 increased in $11.7 \% ; 33 \%$ of the total volume of wastewater were referred to the category of the polluted.

* Corresponding author: gordon1312@mail.ru 


\section{Material and methods}

Domestic literature analysis shows that little attention is paid to environmental risks in scientific research. There is an acute problem of reducing environmental pollution by waste generated from mobile and stationary sources of transport, as part of the urban economy [7]. Motor transport and motor transport facilities in cities create about $70-90 \%$ of all waste and emissions, turning them into centers of major ecological problems [8-10]. At the same time, the development of the engineering and transport structure in the city's life support system, the growing number of cars on the roads and the concomitant increase in the number of stationary objects of the motor transport complex carrying out transportation, refueling, handling, storage, servicing and repair of vehicles, predetermined two clearly expressed and contradictory tendencies. On the one hand, the current level of motorization reflects the technical and economic potential of the society, the ability to meet social needs of the population. On the other hand, the use of vehicles and the functional activities of transport infrastructure have a negative impact on the environment [11, 12]. And this impact is not only significant, but also increasing [13]. This implies that it is useful to study more carefully and to consider in full the impact of environmental factors (air and water pollution) on the demographic situation in the regions and the country as a whole.

The aim of this work is to build a mathematical model of the dynamics and mutual influence of the population and environmental factors - pollution of the atmosphere and surface water bodies - on an urbanized territory.

The model will describe the system whose dynamics is determined by the following properties:

- The environment, surrounding the territory population, receives pollutants, which the population produces;

- Pollutants do not interact with each other and do not form a new harmful substance;

- The population is growing (decreasing) due to self-limiting and irreversible impact of pollutants on it.

\section{Calculation}

The ratio of the mathematical model is based on the following assumptions.

It is known [14] that the growth (decline) of a biological population per unit time is proportional to the first degree of its number:

$$
\Delta \bar{N}=k \bar{N} \Delta \bar{t},
$$

where $\bar{N}-$ is the number of inhabitants; $\Delta \bar{t}-$ is the period of time for which a change in population occurred; $k-$ is the coefficient of proportionality; $\Delta \bar{N}-$ is the population increment.

Substituting in the right side of equation (1) population $\bar{N}$ for multiplication $\bar{N} X(\bar{N})$, where new variable $X(\bar{N})$ is a function, we obtain a complex increase in the population in time. Variable $X$ is called [15] the level of technology, which is proportional to the population number, since it is proportional to the number of "inventors," improving technologies. 
Assuming function $X(\bar{N})$ is linear, i.e. $X(\bar{N})=k_{A} \bar{N}$, we obtain an absolute increment $\Delta \bar{N}(t)$ in the population of the territory in an idealized ("sterile") environment over time interval $\Delta \bar{t}$ :

$$
\Delta \bar{N}(\bar{t})=\bar{a}(\bar{t}) \overline{N^{2}}(\bar{t}) \Delta \bar{t}, \bar{a}=k k_{A}
$$

where $\bar{a}=\bar{a}(\bar{t})$ - is variable increment rate of the population, referred to the square of the population size with dimension, $\left[\right.$ person $/$ person $^{2} \cdot$ time $]$.

Passing in equation (2) to the limit at $\overline{\Delta t} \rightarrow 0$, we obtain a differential equation for the rate of change of the population at time $\bar{t}$ :

$$
\left.\frac{d \bar{N}}{d \bar{t}}=\bar{a}(t) \bar{N}^{2}-\bar{t}\right)
$$

By entering "Malthusian" factors - the quantities of substances polluting the atmosphere $\bar{K}(\bar{t})$ and water bodies $\bar{Z}(\bar{t})$, at given time $\bar{t}$, we get a generalized equation of the population change rate:

$$
\frac{d \bar{N}}{d \bar{t}}=\bar{a}(\bar{t}) \bar{N}^{2}(\bar{t})+\bar{b}(t) \overline{K(t)}+\bar{c}(t) \bar{Z}(\bar{t})
$$

where $\bar{b}=\bar{b}(\bar{t})$ - is variable increment rate of the population, caused by a single amount of substances polluting the atmosphere, with dimension, [person/tons $\cdot$ time $] ; \bar{c}=\bar{c}(\bar{t})-$ is variable increment rate of the population, caused by a single amount of substances polluting water bodies, with dimension $\left[\right.$ person $/ m^{3} \cdot$ time $]$.

It is assumed that parameters $\bar{a}, \bar{b}, \bar{c}$ vary with time for natural reasons or are specially controlled.

Assuming that the pollution has only anthropogenic origin, when changes in the amount of pollution are attributable to a change in the population, increments in the quantities of substances polluting the atmosphere and water bodies for time $\Delta \bar{t}$ takes respectively the form:

$$
\begin{aligned}
\Delta \bar{K}(\bar{t}) & =\bar{d}(\bar{t}) \bar{N}(\bar{t}) \Delta \bar{t}, \\
\Delta \bar{Z}(\bar{t}) & =\bar{g}(\bar{t}) \bar{N}(\bar{t}) \Delta \bar{t}
\end{aligned}
$$

where $\bar{d}=d(\bar{t})$ и $\bar{g}=\bar{g}(t)$ - are variables of increment rate of substances polluting, respectively, the atmosphere and water bodies, caused by one person, with dimensions $[$ tons $/$ person $\cdot$ time $]$ and $\left[\mathrm{m}^{3} /\right.$ person $\cdot$ time $]$. 
Passing to the limits at $\overline{\Delta t} \rightarrow 0$, we obtain the corresponding differential equations for change rates in the quantities of pollutants at time $\bar{t}$ :

$$
\begin{aligned}
& \frac{d \bar{K}}{d \bar{t}}=\bar{d}(\bar{t}) \bar{N}(\bar{t}), \\
& \frac{d \bar{Z}}{\overline{d t}}=\bar{g}(\bar{t}) \bar{N}(\bar{t}) .
\end{aligned}
$$

Thus, we have obtained a non-linear mathematical model of system dynamics for three interdependent functions characterizing a closed urbanized area which consists of three differential equations with rational right-hand sides and variable coefficients. The model allows the kinetic level to describe the evolution of the population of the territory and the volumes of substances that pollute the atmosphere and bodies of water.

Since in equations (4), (7), (8) summands of different dimensions are summed, we reduce the equations to the dimensionless form by entering the following dimensionless variables and parameters:

$$
\begin{gathered}
t=\frac{\bar{t}}{t_{1}}, N=\frac{\bar{N}}{N_{1}}, K=\frac{\bar{K}}{K_{1}}, Z=\frac{\bar{Z}}{Z_{1}}, \\
a=\bar{a} N_{1} t_{1}, b=\frac{\bar{b} K_{1}}{N_{1}} t_{1}, c=\frac{\bar{c} Z_{1}}{N_{1}} t_{1}, d=\frac{\bar{d} N_{1}}{K_{1}} t_{1}, g=\frac{\bar{g} N_{1}}{Z_{1}} t_{1} .
\end{gathered}
$$

We consider time scale $t_{1}$ to be equal to one year, then each unit of dimensionless time $t$ corresponds to the actual duration of one year, that is, $t=1$ means $\bar{t}=1$ year, $t=n$ corresponds to $\bar{t}=n$ years. Accordingly, $N_{1}=N(1), K_{1}=K(1), Z_{1}=Z(1)$ is the population, the amount of air and water bodies' pollutants in the initial (first) year.

Finally, the system of equations (4), (7), (9) takes the form:

$$
\left\{\begin{array}{l}
\frac{d N}{d t}=\left(a_{1} t^{2}+a_{2} t+a_{3}\right) N^{2}(t)+\left(b_{1} t^{2}+b_{2} t+b_{3}\right) K(t)+\left(c_{1} t^{2}+c_{2} t+c_{3}\right) Z(t), \\
\frac{d K}{d t}=\left(d_{1} t^{2}+d_{2} t+d_{3}\right) N(t) \\
\frac{d Z}{d t}=\left(g_{1} t^{2}+g_{2} t+g_{3}\right) N(t) .
\end{array}\right.
$$

The initial conditions in the dimensionless form take the form:

$$
N(1)=1, \quad K(1)=1, Z(1)=1 .
$$

Further, work with the mathematical model is carried out according to the following scheme.

Approximating power functions are constructed for the selected interval $t_{1} \div t_{n}$ by processing the corresponding statistical data (AS Excel). The constructed functions and their derivatives are substituted into equations of the system (10). 
Further, by minimizing the residuals:

$$
\begin{gathered}
R_{1}(t)=a(t) N^{2}(t)+b(t) K(t)+c(t) Z(t)-\frac{d N}{d t} ; R_{2}(t)=d(t) N(t)-\frac{d K}{d t} ; \\
R_{3}(t)=g(t) N(t)-\frac{d Z}{d t}
\end{gathered}
$$

we determine the model parameters $a, b, c, d, g$.

Thus, at this stage, the model parameters are determined by solving an inverse problem using statistical data over a certain time interval.

Further, we solve the direct problem of determining functions $N(t), K(t), Z(t)$ for the forecast period with the found values of the model parameters, using as initial conditions of the process, the values of the functions at the end of the first interval $N\left(t_{n}\right), K\left(t_{n}\right), Z\left(t_{n}\right)$.

The developed model is tested on the example of the Orel region to calculate the dynamics of population and quantities of pollutants with the use of statistical data for a 20year period from 1992 to $2011[1,16]$.

\section{Results and Discussion}

The parameters of the model $a, b, c, d, g(i=1,2,3)$ are determined on the basis of the first actual 11-year data (1992-2002). For this purpose, the trends of factors $N(t), K(t)$, $Z(t)$ are constructed using Excel. Figure 1 shows the correlation fields corresponding to the line and the trend equations, as well as the reliability values of the approximation. Equations with dimensionless variables and constants and their derivatives take the form:

$$
\begin{aligned}
& N(t)=-0.00087 t^{2}+0.0064 t+0.996 ; \quad N^{\prime}(t)=-0.00174 t+0.0064 ; \\
& K(t)=0.007 t^{2}-0.141 t+1.102 ; \quad K^{\prime}(t)=0.014 t-0.141 ; \\
& Z(t)=-0.00035 t^{4}+0.009 t^{3}-0.085 t^{2}+0.33 t+0.74 ; \\
& Z^{\prime}(t)=-0.0014 t^{3}+0.027 t^{2}-0.17 t+0.33 .
\end{aligned}
$$

By substituting the obtained coefficients into the equations of system (10), the direct problem is solved for the next, supposedly forecast period (from 2002 to 2011). The initial conditions are the data for $2002(t=11)$.

Figure 1a shows the graphs of the following functions:

\section{$\mathbf{N}_{\text {er }} \%$}

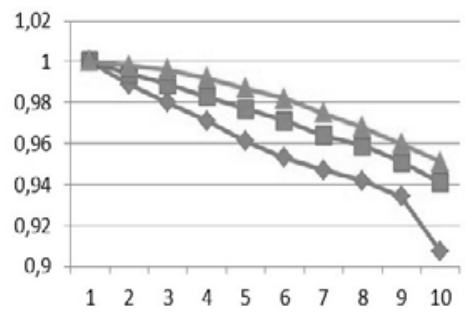

a)

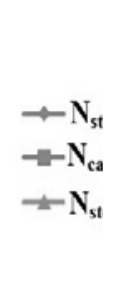

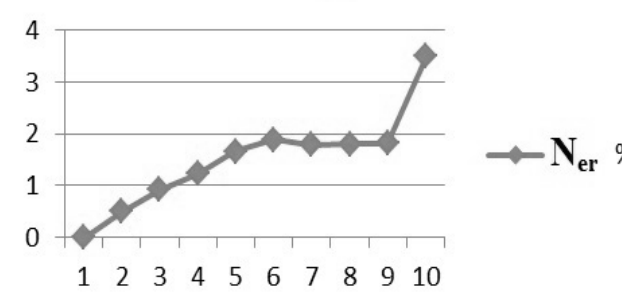

b)

a) - comparison of calculated values of function with statistical ones; b) values of errors in the model calculation

Fig. 1. Dynamics of the population of Orel region in the period $2002-2011$. 
$N_{\text {stat }}(t)$ is the result of processing statistical data on the population of the region for the considered ("forecast") time interval (2002-2011);

$N_{\text {calc }}(t)$ are the results of solving the system of equations of the model (10)-(11);

$N_{\text {ster }}(t)$ is the solution of equation (4), without taking into account the influence of contamination, i.e. at $\bar{b}=\bar{c} \equiv 0$. This function characterizes the dynamics of the population in a "sterile" environment.

Figure $1 \mathrm{~b}$ shows the error values that make up the "forecast" data that is obtained by model (10), with the actual data for the "forecast" period from 2002 to 2011.

$$
N_{\text {calk.er }}=\frac{N_{\text {calk }}-N_{\text {stat }}}{N_{\text {stat }}} \cdot 100 \% .
$$

The calculated trend $N_{\text {calc }}(t)$ predicts a monotonous population decline, which corresponds to known statistical data. Quantitatively, the calculation provides a forecast of high accuracy. Thus, the maximum error in the calculation is $3.5 \%$. Module error increases monotonically in accordance with the forecast range.

Comparison of $N_{\text {calc }}$ and $N_{\text {ster }}$ charts shows that taking into account pollutants in the analysis of population dynamics is justified.

Thus, the paper suggests a method for predicting the ecological and demographic situation of an urbanized territory using a relatively simple nonlinear model with variable coefficients that reflects the interaction of several subsystems (population, pollution of air and water bodies) of an urbanized territory.

The model requires 5 parameters that determine the rate of change of the studied factors, and the initial values of these factors. The procedure for determining the numerical values of the model parameters using statistical data on the investigated factors for a certain period of time is shown.

\section{Conclusion}

The constructed model makes it possible to reproduce the observed trends in the population and the volume of pollutants in a closed urbanized area and make a fairly reliable 5-10 year forecast of the expected tendency changes in these factors and their numerical values.

The efficiency of the model was tested on specific data for the Orel region. The model parameters were calculated taking into account the data for the 11-year period - from 1992 to 2002. "Forecast" was made for the next 10 years $(2002$ - 2011). The results of "forecasting" were compared with the known statistical data for the same years.

The conducted research shows that it is necessary to monitor constantly the natural background and anthropogenic loads on the atmosphere and water bodies to ensure a given level of environmental safety of vehicles and the corresponding urban infrastructure. Tendencies of change in population number, depending on the level of environmental pollution, can be considered an integral indicator of the indirect influence of the motor transport system on human health $[16,17]$. The results of the calculations can be used to support decision making at various management levels.

The disadvantage of the model is the accepted population homogeneity and "depersonalization" of contamination.

The work was carried out within the framework of the basic part of the state task 1.5265.2017/BP (1.5265.2017/8.9). 


\section{References}

1. Regions of Russia. Social and economic indicators of 2012. Moscow: Rosstat. 2012.

2. M. Cole, E. Neumayer, Examing the Impact of Demographic Factors on Air Pollution / Populion and environment, 26, pp. 5-21 (2004)

3. D. Leon, Cities, urbanization and health / Intern. J. Epidemiology, 37(1), pp. 4-8 (2008)

4. A. McMichael, The Urban Enveronment and Health in a World of increasing globalization / Bulletin of the World Health Organization, 78(9), pp. 1117-1126 (2000)

5. S. Galea, N. Freudenberg, D. Vlahov, Cities and population health/ Social science and medicine, 60, pp. 1017-1033 (2005)

6. L. Schell, M. Denham, Environmental Pollution in Urban Environments and Human Biology. / Annual Review of Anthropology, 32, pp. 111-134 (2003)

7. V. Korchagin, Yu. Rizaeva, T. Korchagina, Model search biosphere-compliant operation of the transport socionatureconomic system / World of transport and technological machines, 3(50), pp.130-133 (2015)

8. J. Trofimenko, T. Grigoryeva, I. Avenarius, Estimation of ecological safety of motorcar / Ecology and production in Russia, pp. 18-23 (2007)

9. S. Grishin, E. Kopytov, O. Shchiptsov, Research of transport system influence on ecology of city / Proceedings of the 7 Intern. conf. «Reliability and statistics in transportation and communication - 2007» 24-27 oct. 2007, pp. 2-9, Riga, Latvia, (2007)

10. T. Moiseenko, S. Gashev, Mechanisms of stability and variation of ecosystems in terms of their pollution / Bulletin of the Tyumen state University, 12, pp.17-27, (2011)

11. I. Twardowska, Ecotoxicology, environment safety and sustainable developmentchallenges of the third millennium / Ecotoxicology and environment safety, 58, pp. 3-6, (2004)

12. L. Stavnikova, R. Stepen, Ecological risk assessment of industrial and vechicular emissions / Bulletin of the Tyumen state University, 12, pp.175-181, (2011)

13. V. Fedorov, A. Pozdnyakov, Ensuring the safety of living environment is an urgent matter of time. Proceedings of the International scientific-practical conference «Biosphere-compatible cities and settlements», pp. 64-69, Bryansk, Russia, (2012)

14. Yu. Slovohotov, Analogues of phase transitions in economics and demography. Computer Studies and Modeling, 2, pp.76-91, (2010)

15. A. Korotaev, Compact mathematical macromodel of technical, economic and demographic growth World-systems. History and Synergetics: Mathematical Modeling of Social Dynamics. Moscow: Komkniga, pp. 46-48, (2005)

16. O. Ivaschuk, Increase of ecological safety of motor transport of the region on the basis of monitoring system using intellectual technologies. Orel: Publishing house OrelGAU, 244 p., (2008)

17. V. Vasil'yeva, A. Katunin, Y. Charskiy, D. Kozhin, D. Alokminskiy, Reducing the level of emissions of motor vehicles by determining the rational operating modes of traffic lights at a crossroads /The world of transport and technological machines, $\mathbf{1}$ (56), pp. 119-125, (2017) 